\title{
Lateral pectoral nerve transfer for spinal accessory nerve injury
}

\author{
Andrés A. Maldonado, MD, PhD, and Robert J. Spinner, MD \\ Department of Neurologic Surgery, Mayo Clinic, Rochester, Minnesota
}

Spinal accessory nerve (SAN) injury results in loss of motor function of the trapezius muscle and leads to severe shoulder problems. Primary end-to-end or graft repair is usually the standard treatment. The authors present 2 patients who presented late (8 and 10 months) after their SAN injuries, in whom a lateral pectoral nerve transfer to the SAN was performed successfully using a supraclavicular approach.

http://thejns.org/doi/abs/10.3171/2016.5.SPINE151458

KEY WORDS spinal accessory nerve; cranial nerve Xl; lateral pectoral nerve; nerve injury; nerve transfer; neurotization; technique

0 PINAL accessory nerve (SAN) injury results in loss of motor function of the trapezius muscle and leads to weakness of the shoulder in abduction, winging of the scapula, drooping of the shoulder, and pain and stiffness in the shoulder girdle. The majority of the cases of SAN injury occur in the posterior triangle of the neck. When the SAN is transected or a nonrecovering neuromain-continuity is observed, the standard treatment would include a primary end-to-end or graft repair. ${ }^{2}$ Nerve transfers may be considered, especially in cases of proximal injury or delayed presentation. In this paper we present 2 patients with long-standing SAN injury in whom a lateral pectoral nerve (LPN) was transferred to the SAN successfully.

\section{Illustrative Cases}

After obtaining institutional review board approval, a retrospective review of 2 cases with LPN transfer to the SAN was performed.

\section{Case 1}

A 51-year-old man was referred for bilateral SAN lesions lasting 8 months. He underwent treatment for a Grade 3 squamous cell carcinoma of the left tongue after prior resection, chemotherapy, and radiation therapy. The left SAN was intentionally transected due to the proximity of the cancer to it. The right SAN was identified, mobilized, and preserved as part of the lymph node dissection. Postoperatively, the patient experienced severely impaired active shoulder motion bilaterally, with shoulder pain. On physical examination, the patient showed bilateral trapezius muscle atrophy and moderate left scapula winging. Left arm abduction was limited to approximately $45^{\circ}$ and his right arm presented with a full arc of motion (Fig. 1A). Electrophysiological studies showed evidence of SAN neuropathy on the left greater than on the right, distal to the innervation of the sternocleidomastoid (SCM) muscle.

\section{Case 2}

A 22-year-old woman came for evaluation of her left SAN lesion. Two years previously the patient had experienced daily headaches and was found to have papilledema. She was diagnosed with occlusion of the right transverse sinus and left jugular vein and was treated with balloon angioplasty of both lesions. One year later, she noted some difficulty lifting her left arm and shoulder, with little pain. Compression of the left SAN upon the stent against the styloid process was diagnosed. Although her first surgeon 


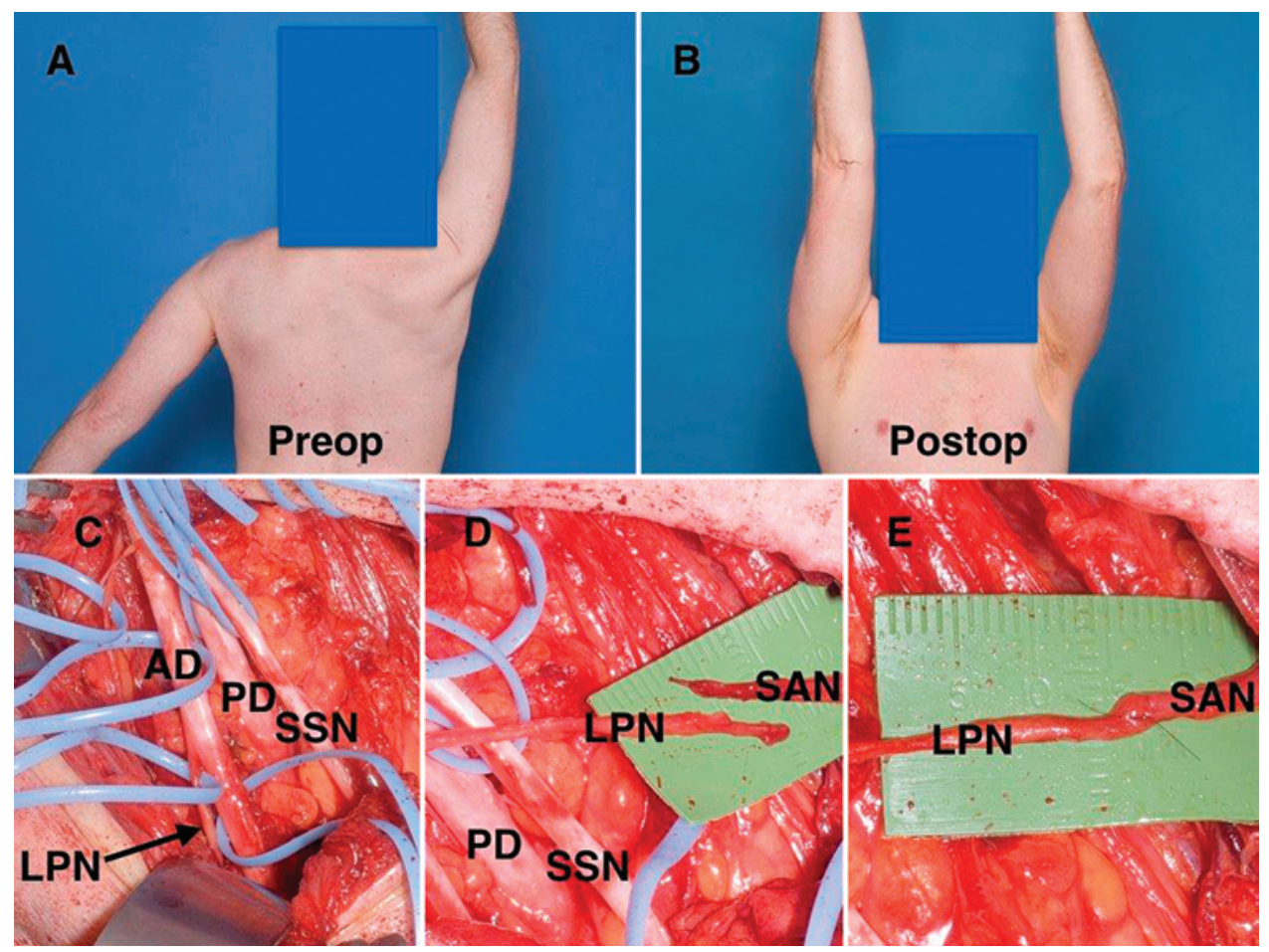

FIG. 1. Case 1. A: Preoperative photograph obtained 8 months after a bilateral SAN injury. Left arm abduction was limited to approximately $45^{\circ}$. B: Follow-up photograph taken 6 years after surgery. C: Dissection of the left LPN coming from the anterior division (AD) of the superior trunk. D: The LPN was swung upward and could be coapted directly to the SAN distal stump. E: The LPN repair to the distal stump of the SAN is performed without tension. $A D=$ anterior division of the upper trunk; $P D=$ posterior division of upper trunk; SSN = suprascapular nerve.

considered a styloidectomy, the final decision made was to avoid another surgery. Ten months after the onset of the arm weakness, the patient presented to our clinic. On physical examination, she was found to have left scapular winging and arm abduction limited to approximately $70^{\circ}$. Electrophysiological studies showed a severe and complete proximal left SAN neuropathy without evidence of reinnervation of the trapezius and SCM muscles.

\section{Surgical Technique}

The same technique was used in both patients (Fig. 1C-E and Fig. 2D-F). Through a single 4-cm low transverse supraclavicular incision, the nerve transfer was accomplished (Fig. 3). Subplatysmal flaps were created. The distal SAN was identified along the medial border of the trapezius muscle and was traced proximally toward its distal stump and then mobilized distally. The upper trunk, its anterior and posterior divisions, and the suprascapular nerve were then identified and mobilized. The clavicle was mobilized in umbilical tape and was retracted caudally. The LPN coming from the anterior division was identified and stimulated. The LPN was dissected distally in the retroclavicular space. This nerve stimulated the clavicular portion of the pectoralis major muscle. The LPN was transected distally and was swung upward to coapt directly to the SAN distal stump. A favorable size match for the transfer was found (the LPN had a comparable diameter to the SAN; Figs. 1E and 2F). Nerve repair was performed without tension, using a standard microsurgical technique.

Postoperatively the arm was maintained in a sling for 3 weeks. At that time, the patients started a physical therapy program that emphasized passive and active shoulder motion and strengthening exercises. Three to 6 months after surgery, when there was evidence of early reinnervation, the rehabilitation program included standard protocols after nerve transfers, ${ }^{6}$ which emphasized motor reeducation (i.e., how to recruit the newly reinnervated muscle) and restoration of muscle balance.

\section{Results \\ Case 1}

Follow-up of this patient was conducted 5 months and 6 years after surgery (Fig. 1B). After 5 months electrophysiological studies showed chronic bilateral accessory neuropathies with evidence of ongoing reinnervation and clear improvement compared with the prior study. Some trapezius muscle bulk was present. The patient had no shoulder pain. After 6 years the patient had regained useful overhead shoulder motion. Shoulder abduction was $160^{\circ}$. He still had some atrophy in his left trapezius muscle. He fired his pectoralis major muscle to activate his shoulder abduction. Mild left scapula winging was present. His right side improved spontaneously. 

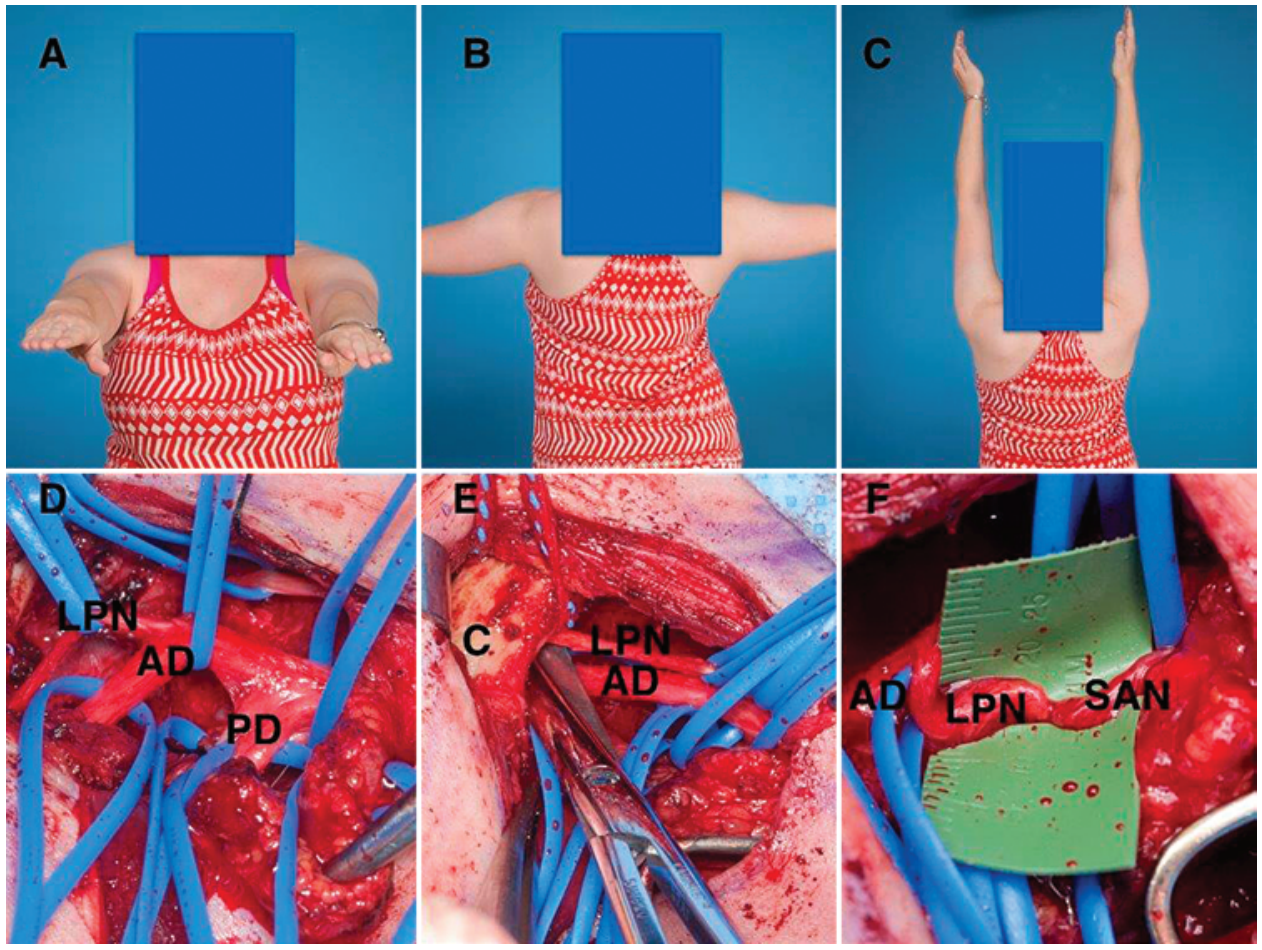

FIG. 2. Case 2. A-C: Postoperative photographs taken 2.5 years after surgery. D: Intraoperative dissection of the left LPN. E: The LPN is observed before being cut beneath the clavicle ( $\mathrm{C}=$ clavicle). F: The LPN and SAN were mobilized for direct coaptation. Note the larger size of the LPN compared with the SAN. AD = anterior division of upper trunk; PD = posterior division of upper trunk.

\section{Case 2}

Follow-up of this patient was conducted at 6 months and 2.5 years after the reconstruction (Fig. 2A-C). Electrophysiological studies performed 6 months after surgery showed evidence of reinnervation and improvement from the prior study. Two and a half years after surgery the upper trapezius function was good and muscle bulk was present. She regained some function in the lower trapezius as well, although there was some atrophy. Her SCM muscle remained atrophic. She was able to abduct her arm fully without pain, although she tended to compensate to some degree. Mild winging of the scapula was still present.

\section{Discussion}

In this paper we present a novel nerve transfer using the LPN that may be considered in patients with chronic SAN injury, particularly in those in whom there are other poor prognostic factors for nerve recovery: prior radiation treatment in the first patient, and a proximal level injury in the second one. A second potential indication would be for patients with previous extensive neck injury/dissection in whom a proximal accessory nerve could be difficult to identify. Other nerve transfers have been described previously. One patient was treated using the medial pectoral branches through an infraclavicular approach combined with a supraclavicular incision. ${ }^{7}$ A second patient was treated using a fascicle from the C-7 middle trunk ${ }^{5}$ through a supraclavicular approach.
Our approach using the LPN is based on anatomical studies, ${ }^{1,4}$ which showed that the pectoral nerves arise from the divisions in the retroclavicular space rather than at the cord level. Thus, we were able to complete the entire procedure through a straightforward supraclavicular approach. We believe this approach has 3 main advantages compared with these other reported techniques: 1) the single incision for the LPN and SAN dissection avoids a separate infraclavicular incision and dissection, yet still allows a direct nerve repair without nerve graft; 2 ) the LPN (the superior pectoral branch, according to the description by Aszmann et al. $\left.{ }^{1}\right)$ has more myelinated nerve fibers (2637 $\pm 839)$ than the medial pectoral branch $(1784 \pm 445)$; in comparison, the C-7 pectoral branch contribution has 1455 \pm 191 myelinated fibers ${ }^{1}$ and the SAN $1300 ;{ }^{8}$ and 3 ) use of the LPN in this fashion allows adequate length for the nerve transfer without interfascicular dissection to identify or mobilize a C-7 fascicle. In fact, additional length of the LPN donor could be easily obtained through the same skin incision by working below the clavicle as necessary: dividing the subclavius muscle, mobilizing the clavicle cephalad, and dissecting the LPN branch distally as necessary. In our 2 patients with chronic injury as well as prior radiation exposure or a proximal lesion, a nerve transfer performed in normal tissue planes appeared preferable to lengthier nerve grafting through previous scarring.

Secondary reconstruction using tendon transfers has also been described. This can be considered as a salvage procedure when trapezius muscle reinnervation is not pos- 


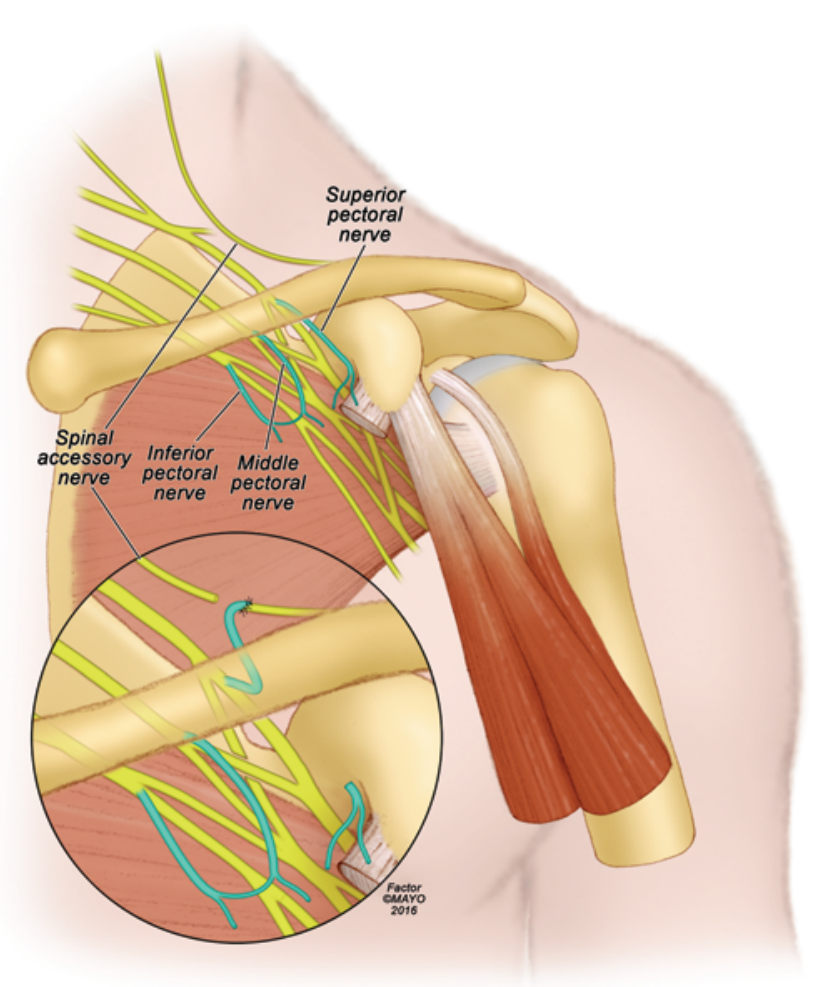

FIG. 3. Schematic illustration of the left LPN transfer to the SAN. Note that the pectoral nerves arise from the divisions in the retroclavicular space rather than at the cord level, as described by Aszmann et al. ${ }^{1}$ Used with permission of the Mayo Foundation for Medical Education and Research. All rights reserved.

sible (SAN long-term injuries) or if a nerve procedure has failed. The Eden-Lange procedure to reconstruct the trapezius, described by Eden and verified by Lange in the first half of the 20th century, entails transfer of the levator scapulae to the spine of the scapula, and the rhomboid minor and major to the midbody of the scapula. Recently a variation of this procedure has been reported with some improvement in outcomes. ${ }^{3}$ In general, these tendon transfers have more morbidity and require more rehabilitation than our described nerve transfer.

The nerve transfer we describe does not target the SCM muscle as it is performed distal to the SCM nerve branch. We believe that the distal nerve transfer is advantageous in accelerating the muscle reinnervation, especially in longterm SAN injuries. Restoring function of the SCM muscle is not a priority (it should be noted that this muscle is frequently resected or divided without major consequence). After our nerve transfer, patients had to readapt and learn to activate the trapezius (by contracting the pectoralis major) to achieve shoulder abduction, in addition to suprascapular and deltoid muscle. The patients were able to learn this readily. The nerve transfer improved scapular stability (range of motion and winging) and decreased pain. This paper describes LPN transfer to the SAN through a supraclavicular approach, and we believe that this technique can be considered in select cases of chronic SAN injury.

\section{References}

1. Aszmann OC, Rab M, Kamolz L, Frey M: The anatomy of the pectoral nerves and their significance in brachial plexus reconstruction. J Hand Surg Am 25:942-947, 2000

2. Bradley PJ, Ferlito A, Silver CE, Takes RP, Woolgar JA, Strojan P, et al: Neck treatment and shoulder morbidity: still a challenge. Head Neck 33:1060-1067, 2011

3. Elhassan BT, Wagner ER: Outcome of triple-tendon transfer, an Eden-Lange variant, to reconstruct trapezius paralysis. J Shoulder Elbow Surg 24:1307-1313, 2015

4. Johnson D: Pectoral girdle, shoulder region and axilla, in Standring S (ed): Anatomy: The Anatomical Basis of Clinical Practice, ed 40. Edinburgh: Churchill Livingstone/ Elsevier, 2008, pp 791-822

5. Magill CK, Moore AM, Mackinnon SE: Same modality nerve reconstruction for accessory nerve injuries. Otolaryngol Head Neck Surg 139:854-856, 2008

6. Novak CB: Rehabilitation following motor nerve transfers. Hand Clin 24:417-423, vi, 2008

7. Novak CB, Mackinnon SE: Treatment of a proximal accessory nerve injury with nerve transfer. Laryngoscope 114:1482-1484, 2004

8. Schreiber JJ, Byun DJ, Khair MM, Rosenblatt L, Lee SK, Wolfe SW: Optimal axon counts for brachial plexus nerve transfers to restore elbow flexion. Plast Reconstr Surg 135:135e-141e, 2015

\section{Disclosures}

The authors report no conflict of interest concerning the materials or methods used in this study or the findings specified in this paper.

\section{Author Contributions}

Conception and design: Spinner. Acquisition of data: Maldonado. Analysis and interpretation of data: Maldonado. Drafting the article: Maldonado. Critically revising the article: both authors. Reviewed submitted version of manuscript: both authors. Approved the final version of the manuscript on behalf of both authors: Spinner. Study supervision: Spinner.

\section{Correspondence}

Robert J. Spinner, Department of Neurologic Surgery, Mayo Clinic, Gonda 8-214, Rochester, MN 55905. email: spinner. robert@mayo.edu. 